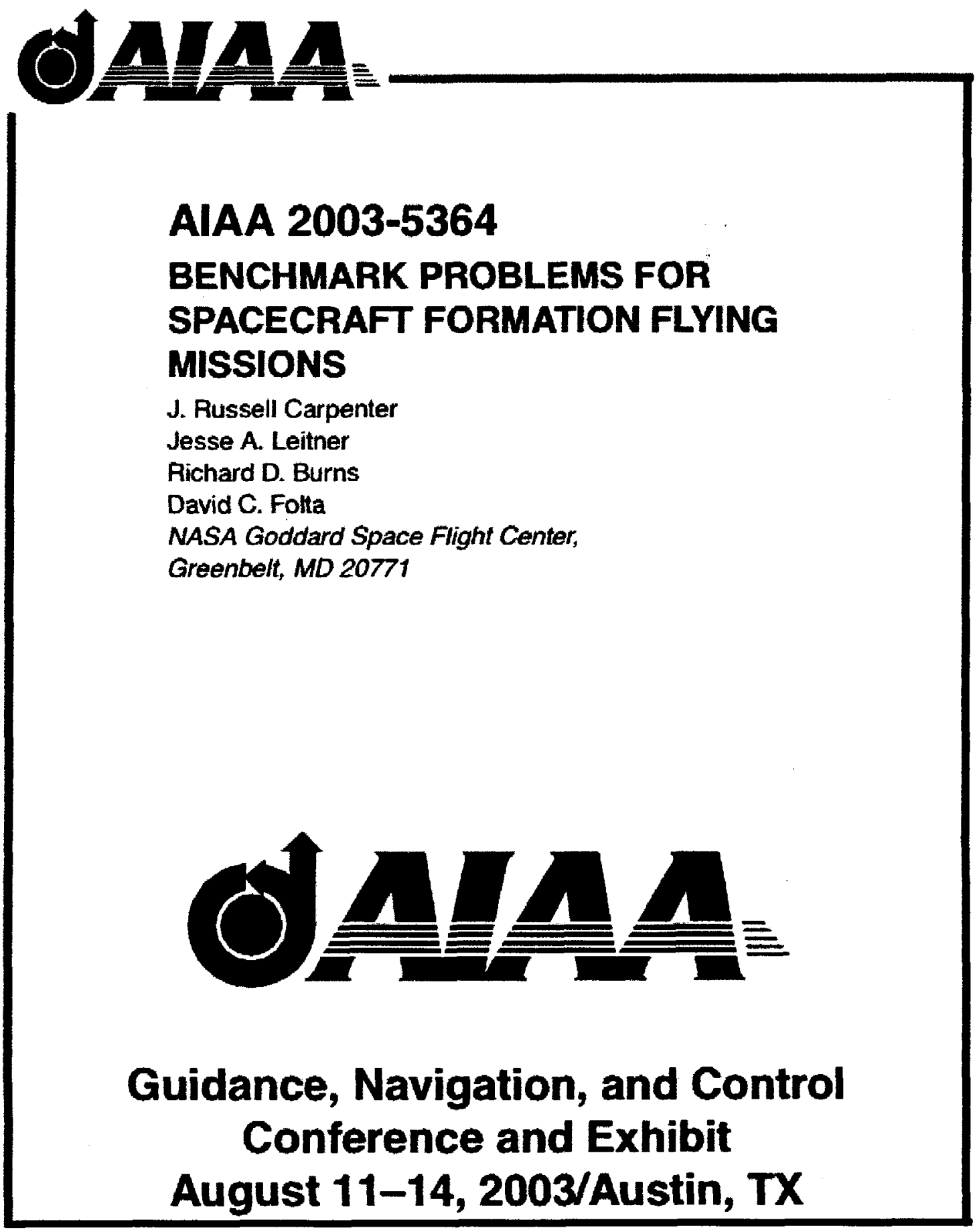

For permission to copy or republish, contact the American mstitute of Aeronautics and Astronautics 1801 Aloxander Bell Drive, Suite 500, Reston, VA 20191-4344 


\title{
BENCHMARK PROBLEMS FOR SPACECRAFT FORMATION FLYING MISSIONS
}

\author{
J. Russell Carpenter", Jesse A. Leitner ${ }^{\dagger}$, David C. Folta, Richard D. Burns ${ }^{\dagger}$ \\ NASA Goddard Space Flight Center, Greenbelt, MD 20771
}

\section{Abstract}

To provide high-level focus to distributed space system flight dynamics and control research, several benchmark problems are suggested. These problems are not specific to any current or proposed mission, but instead are intended to capture high-level features that would be generic to many similar missions.

\section{Introduction}

Collection of information through a cluster of individual sensors or through sensors distributed over multiple formation flying vehicles can provide substantial benefits. Distributed space systems (DSS) enable higher resolution imagery and interferometry, robust and redundant fault-tolerant spacecraft system architectures, and complex networks dispersed over clusters of satellites in space ${ }^{1}$. As DSS technologies have matured in recent years, various groups and agencies are flying and proposing a large number of DSS missions ${ }^{2}$. These missions and mission concepts cover a wide range of flight environments, from low Earth orbit (LEO) to deep space, and requirements regimes from relative control precisions of nanometers to kilometers, over baselines of tens of meters to millions of kilometers. The more challenging mission concepts envision launches in the mid- to late-2010s, but several ambitious missions are proposed and funded for launch in the 2007-2015 timeframe, and are a primary focus of much of the recent interest in spacecraft formation flying.

Due to the wide variety of ideas for DSS missions, researchers working in this area have sometimes had difficulty in identifying candidate problems to which they should apply their innovations. Researchers have also sometimes had difficulties in making relevant comparisons between algorithms and technologies when they are applied in different circumstances. This paper attempts to address these concerns by suggesting some relevant benchmark problem descriptions that cover a range of the types of missions that are of interest to NASA over the next couple of decades. These problems are not specific to any current or proposed mission, but instead are intended to capture high-level features that would be generic to many similar missions. In particular, however, this paper focuses on the problem of formation flying spacecraft. This focus bounds into the fundamental foundation for formation flying, which is the understanding, harnessing, and exploitation of the dynamics of relative motion.

\section{Benchmark Problems}

The subsections below present three benchmark problems, which are labeled by their orbital flight regimes: low Earth orbit; highly elliptical Earth orbit; and libration point orbit. The problem descriptions intentionally avoid specifying design details such as mass and area, as well as specific capabilities such as thrust resolution, navigation accuracy, or control precision.

In addition to the requirements each problem contains, overall goals are the minimization of resource usage (e.g. fuel, power), launch mass, and control, guidance, or navigation error depending on the type of investigation. Potential constraints on the system, which may govern the formation control design, include fuel budget, maneuver frequency, thrust magnitude, and navigation accuracy. We will focus on the formation problem, not on a collection of individual

\footnotetext{
* Aerospace Engineer, Flight Dynamics Analysis Branch, Mission Engineering and Systems Analysis Division. Senior Member, AIAA.

${ }^{\dagger}$ Aerospace Engineer, Guidance, Navigation, and Control Systems Engineering Branch, Mission Engineering and Systems Analysis Division. Senior Member, AIAA.
} 
spacecraft. Where the problems specify numerical performance requirements, these should be met with a probability of $99 \%$, with $95 \%$ confidence. Where the problems specify requirements of more than one type, e.g. both relative position and pointing, or control and knowledge, researchers are encouraged to seek solutions that meet any or all of the problems' specifications. While system-level analysis is essential for looking at the broad problem of formation flying spacecraft, it is important that detailed research and development work on the component technology areas (relative navigation, formation control, formation design, etc.) does not become washed out in high-level architecture development. Thus we propose to form an integrated team across the community across all of the internal disciplines and integrated with the mission development teams and principle investigators.

Unless otherwise noted, all spacecraft in each formation should be nominally identical in their physical

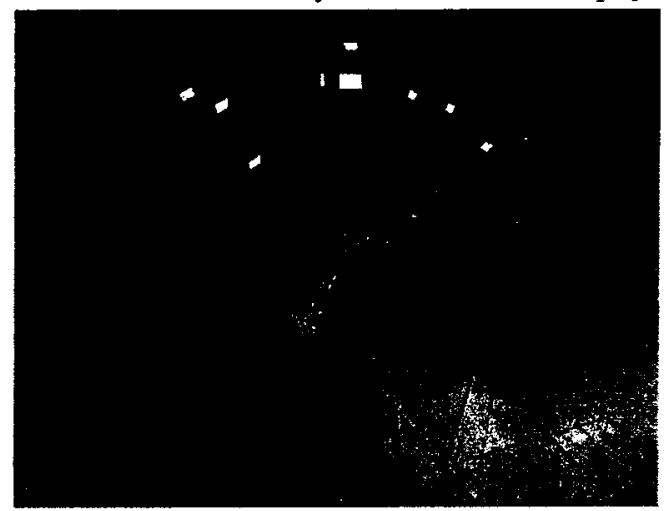

Figure 1: Concept for a proposed NASA LEO DSS mission.

properties, but researchers may wish to consider variations of $5 \%$ as a source of modeling error and much more substantial variations in parameters such as ballistic coefficient because of varying orientations within a formation in a drag environment. Other than the constraints listed here, one may assume any kind of sensor, actuator, and plant properties and capabilities that should be available in the 2005-2015 timeframe.

\section{Low Earth Orbit}

Figure 1 illustrates a proposed NASA mission concept that shares some characteristics with the low Earth orbit (LEO) benchmark problem. The benchmark formation is defined with respect to a reference trajectory, which follows a near-circular, sunsynchronous orbit with a nominal altitude of $400 \mathrm{~km}$.
A nominal set of mean orbital elements are presented in Table 1 , where $a$ is the semi-major axis, $e$ is the eccentricity, $i$ is the orbital inclination, $\Omega$ is the right ascension of the ascending node, $\omega$ is the argument of perigee, and $f$ is the true anomaly. The term mean elements indicates that short period effects have been removed either analytically or through some numerical averaging process.

Table 1: LEO reference trajectory mean elements

\begin{tabular}{ccc}
\hline Description & Value & Units \\
\hline Epoch & 5 Oct 2001 0h 0m 0s & UT0 \\
$a$ & 6778 & $\mathrm{~km}$ \\
$e$ & $1.000 \times 10^{-3}$ & none \\
$i$ & 97.03 & $\mathrm{deg}$ \\
$\Omega$ & 13.71 & $\mathrm{deg}$ \\
$\omega$ & 0 & $\mathrm{deg}$ \\
$f$ & 0 & $\mathrm{deg}$ \\
\hline
\end{tabular}

No spacecraft exists at the reference point. There are six, 3-axis stabilized spacecraft. Three are equally spaced in each of two oppositely inclined "projected circular" formations $500 \mathrm{~m}$ in diameter. That is, when projected into the along-track/cross-track plane of the reference orbit, the paths of relative motion about the reference are circles in the linearized relative motion approximation (Hill's Equations). This relative motion can be achieved through a combination of differences in the orbital elements from the reference trajectory. These orbital element differences are dependent on the diameter of the formation and the phase of the spacecraft within the formation. Sabol et $\mathrm{al}^{4}$ have provided relationships for initializing projected circular formations and noted that the semi-major axes should be matched exactly in order for the formation to be stable under Keplerian motion.

The phase within the formation will determine the required inclination and ascending node offsets from the reference. The inclusion of three satellites in each formation necessarily implies that at least one formation member will have an inclination offset from the other members, which establishes differential nodal and in-plane drifts caused by the $\mathrm{J}_{2}$ geopotential term. Schaub and Alfriend ${ }^{5}$ have provided relationships to manipulate semi-major axis, eccentricity, and inclination offsets in order to null the differential nodal and in-plane drift, but this method encounters singularities at polar inclinations and circular orbits. Alfriend ${ }^{6}$ has proposed a relaxation of these constraints near circular and polar orbits along with a novel method for balancing fuel expenditure 
within a similar formation. The relative positions of the spacecraft must be controlled with respect to their desired relative trajectories to within $5 \mathrm{~m}$ or better. The spacecraft should be capable of pointing to a common target on the surface of the earth to within 0.1 degrees. The nominal mission duration is two years.

\section{Highly Elliptic Orbit}

Figure 2 depicts a proposed NASA mission ${ }^{7}$ that shares come characteristics with the highly elliptical orbit (HEO) benchmark problem. The average position of the HEO benchmark formation follows a 1.2 by 18 Earth radii (ER) orbit, lying approximately 5 degrees above the ecliptic plane, where the initial line of apsides is parallel to the direction to the sun, and apogee is opposite the sun. Table 2 lists a nominal set of mean elements, following the convention described above.

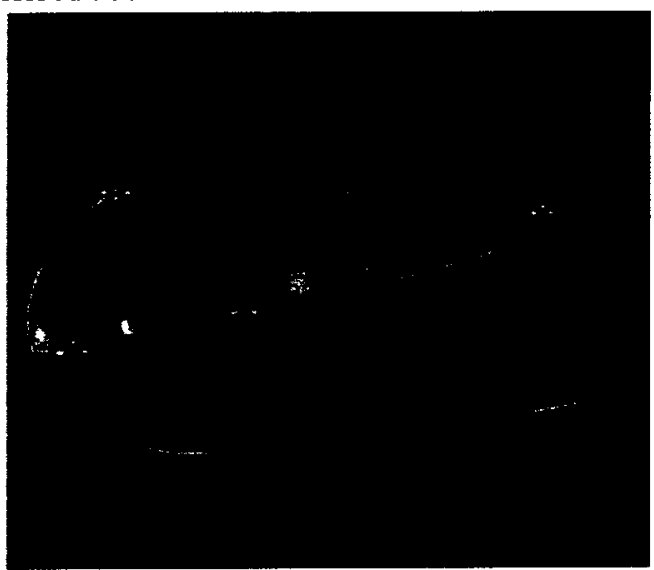

Figure 2: Proposed NASA HEO mission. This mission has four phases: an initial HEO of $1.2 \times 12 \mathrm{ER}$, approximately in the ecliptic plane; a $1.2 \times 30$ ER phasing orbit; a double lunar swingby; and a final $\mathrm{HEO}$ of $10 \times 40 \mathrm{ER}$ in a near polar orbit.

Table 2: HEO reference trajectory mean elements

\begin{tabular}{ccc}
\hline Description & Value & Units \\
\hline Epoch & 21 Jun 2009 0h 0m 0s & UT0 \\
Ref. Frame & Earth Equator, MJ2000 & \\
$a$ & 61277 & $\mathrm{~km}$ \\
$e$ & 0.875 & none \\
$i$ & 18.5 & deg \\
$\Omega$ & 0 & deg \\
$\omega$ & 90 & deg \\
$f$ & 0 & deg \\
\hline
\end{tabular}

No spacecraft exists on the reference orbit. There are four spin-stabilized spacecraft that must form a $10 \mathrm{~km}$ regular tetrahedron at apogee, with arbitrary orientation. A tetrahedral configuration need not be maintained at any other point in the orbit. The desired relative motion can be achieved through a combination of differences in a subset of the orbital elements from the reference trajectory, excluding semimajor axis, which as in the LEO case, must be matched exactly. These orbital element differences determine the phasing of the individual spacecraft in the formation, which determines the orientation of the tetrahedron, as well as the formation's relative motion throughout the remainder of the orbit. With respect to relative motion throughout the remainder of the orbit, it is desirable that variations in the volume of the tetrahedron, and in the ratio of the maximum to minimum interspacecraft ranges, be minimized while within 30 degrees of apogee, Also, close approaches of $1 \mathrm{~km}$ or less should be strictly avoided throughout the orbit. Mailhe, et al. ${ }^{8}$, compare several approaches that address some of these requirements.

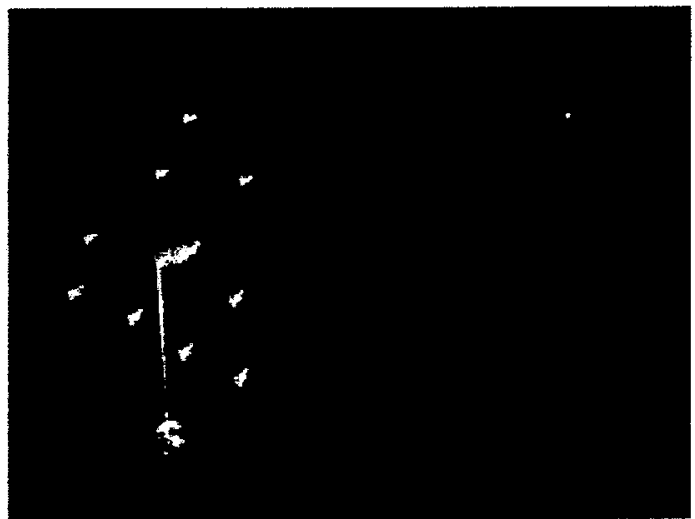

Figure 3: Concept for NASA libration point mission.

Each spacecraft's spin rate is $20 \pm 2$ RPM, and the spin axis is perpendicular to the ecliptic plane, to within 1 degree. The spacecrafts' relative positions should be controlled at apogee to within $10 \%$ of the separation, but are not constrained throughout the remainder of the orbit, except that they must maintain $1 \mathrm{~km}$ minimum separation constraint. The nominal mission duration is two years.

\section{Libration Point}

The formation follows a medium lissajous orbit about the trans-terrestrial, now commonly known as "L2," co-linear libration point of the Sun-Earth/Moon system, with $y$-, or transverse, amplitude of approximately $300,000 \mathrm{~km}$ and $z$-, or normal, amplitude equal to or less than the $y$-amplitude. There are $203-$ axis-stabilized spacecraft, each a subaperture along an aspherical surface with a $250 \mathrm{~m}$ radius. The su- 
bapertures are distributed over the asphere in an arbitrary configuration so as to produce a large number of internal baselines for a sparse primary telescope aperture. A single spacecraft is located $100 \mathrm{~km}$ away at the focus, along the line of sight to the science target, such that the whole configuration forms a distributed Fizeau interferometer. Figure 3 illustrates the concept for a proposed NASA mission that share some similarities with this problem ${ }^{9}$.

There are two modes of operation:

- A science mode, in which the aperture must "stare" in an inertially fixed direction for up to a month, with relative positions controlled to within $1 \mathrm{~cm}$. While staring, the formation must rotate about the line of sight to the target at least once per week, and the internal baselines of the aperture must vary somewhat arbitrarily during the rotation. Pointing requirements, absolute and relative, in this mode are 1 arcsec.

- A maneuver mode, in which the line of sight of the aperture must slew on the order of 20 degrees per day to acquire a new science target. In this mode, the only attitude determination requirement is the need to point sensors and actuators with enough accuracy to efficiently perform the maneuver.

- The line-of-sight of the formation (the line along the focal length) must be maintain relative to an inertial source on the order of the imaging resolution which is approximately 10 micro-arcseconds.

The nominal mission duration is 12 years.

\section{Summary}

This paper has described, at a high level, three benchmark problems covering a range of the types of distributed spacecraft missions that NASA and other agencies hope to accomplish over the next couple of decades. The requirements herein are characteristic of similar missions but in many cases are more or less stringent than those in the actual mission concepts that they represent. The purpose of these problems is to foster better collaboration and comparison of results among the many researchers engaged in the study of guidance, navigation, and control issues for spacecraft formation flying.

\section{Acknowledgment}

This paper benefited from conversations with Steven Hughes.

\section{References}

1. Leitner, J., et al. "Formation Flight in Space: Distributed Spacecraft Systems Develop New GPS Capabilities," GPS World, Feb 2001.

2. http://gsfctechnology.gsfc.nasa.gov/dssmissionli st.htm

3. Vallado, D., Fundamentals of Astrodynamics and Applications, Microcosm Press, El Segundo, CA, 2001.

4. Sabol, C., et al, "Satellite Formation Flying Design and Evolution," Journal of Spacecraft and Rockets, v. 38, no. 2, Mar-Apr 2001.

5. Schaub, $\mathrm{H}$. and Alfriend, $\mathrm{K}$., " $\mathrm{J}_{2}$ Invariant Relative Orbits for Spacecraft Formations"' Celestial Mechanics and Dynamical Astronomy, v. 79, p. 77-95, 2001.

6. Alfriend, K., et al, "Formation Flying Satellites: Control by an Astrodynamicist," Celestial Mechanics and Dynamical Astronomy, v. 81, p. 5762, 2001.

7. http://mms.gsfc.nasa.gov

8. Mailhe, L., et al., "Formation Flying in Highly Elliptical Orbits: Initializing the Formation," CNES 15th International Symposium on Space Flight Dynamics, June 26-30, 2000, Biarritz, France.

9. http://hires.gsfc.nasa.gov/ -si 